\title{
High-Performance Sensors Based on Molybdenum Disulfide Thin
}

\author{
Films Lee, K, Gatensby, R, McEvoy, N, Hallam, T, Duesberg, GS,
}

\author{
Keywords: \\ microstructures; molecular electronics; sensors; thin films; charge transport
}

\begin{abstract}
Two-dimensional (2D) layered materials are currently being studied as candidates for future electronic devices. Recently, it has been shown that layered transition-metal dichalcogenides (TMDs), such as molybdenum disulfide $\left(\mathrm{MoS}_{2}\right)$, have, unlike graphene, a bandgap sufficient for device applications.[1-6] According to recent reports on single- or a few-layered $\mathrm{MoS}_{2}$ crystals, they offer excellent electronic performance in a top gate structure with outstanding on-off ratios, which are comparable to those of graphene nanoribbons.[3] Also, these structural analogues of graphene potentially promise ultra-high sensitivities for detection of environmental molecules due to their high surface-to-volume ratio. Electronic sensors based on chemical field-effect transistor (ChemFET) or chemical resistor (Chemiresitor) configurations change their conductivities when analytes are adsorbed on the active channel, with the magnitude of the change dependent on the electron donating or withdrawing strength of the analyte.
\end{abstract}

The use of nanomaterials as the channel material is beneficial because of their large surface area. In the case of monolayer materials or nanotubes, the whole surface is theoretically exposed to external influences. However, there are certain limitations, such as low sensitivity to analytes, which have low affinity or binding energy, or lack of selectivity. Thus functionalization is necessary to sensitize the surface or to engender selectivity. In the case of inorganic 2D materials, such as transition-metal oxides (TMOs) or chalcogenides (TMCs), an in-built selectivity is present due to their varying affinity for different analytes. Initial results have revealed that $\mathrm{MoS}_{2}$ layers are also extremely sensitive to $\mathrm{NO}_{x}[7]$ and $\mathrm{NH}_{3} \cdot[8]$

However, to date most studies of layered 2D materials have been based on materials produced by mechanical cleaving which is a serendipitous method, thus its throughput is seriously limited. For commercialization, scalable, low-cost, high-yield production of active channels and compatibility with conventional semiconductor fabrication processes are essential. For this reason, as in the case of graphene, many researchers have sought to move beyond the cleaving technique and towards large quantity production of $\mathrm{MoS}_{2}$ nanosheets. [2] It has been demonstrated that the electrical performance of liquid exfoliated flakes match those of mechanically cleaved ones,[9] however residual solvents and impurity contamination, as well as flake to flake contacts, deteriorate electrical performance when these are assembled into thin films.

Chemical and physical vapor deposition (CVD, PVD) of 2D materials are considered the methods of choice for synthesis of high quality films, on a large scale, in a reproducible manner. In this study, we investigate the sensor performance of scalable $\mathrm{MoS}_{2}$ films synthesized by sulfurization of sputtered Mo thin-films, similar to the method recently described by Zhan et al.[10] We were able to yield highly homogeneous and structured $\mathrm{MoS}_{2}$ patterns, which were directly contactable by electrode deposition with shadow masks. This two-step process flow yields highly sensitive sensors for the detection of ammonia $\left(\mathrm{NH}_{3}\right)$ at sub-ppm (parts-per-million) levels. The study shows that layered $2 \mathrm{D}$ materials are fully compatible with semiconductor fabrication technology and can lead to cheap and high performance devices.

The pristine Mo layers sputtered were nominally $10 \mathrm{~nm}$ thick as determined by the quartz crystal balance in the sputter tool. After sulfurization, the thickness of the layers nearly doubles to an approximately $20 \mathrm{~nm}$ thick $\mathrm{MoS}_{2}$ film, as determined by atomic force microscopy (AFM) on a step edge, as shown in the inset of Figure $1 \mathrm{~b}$. The film is uniform over large areas and the roughness of the film is $2.5 \mathrm{~nm}$ root-mean-square (RMS). Raman spectra of our $\mathrm{MoS}_{2}$ film are shown in Figure 1c. Two strong peaks are observed at $382.5 \mathrm{~cm}^{-1}$ and 408 $\mathrm{cm}^{-1}$, these are characteristic of $\mathrm{MoS}_{2}$ and correspond to $E^{1}{ }_{2 \mathrm{~g}}$ and $A_{1 \mathrm{~g}}$ vibrational modes, respectively.[11] Additional contributions in the range $450-460 \mathrm{~cm}^{-1}$ can be attributed to the $2 L A(M)$ and $A_{2 u}$ modes. 12] We mapped the intensity of the $A_{1 \mathrm{~g}}$ peak sum over a $10 \mu \mathrm{m} \times 10$ $\mu \mathrm{m}$ area, as shown in the inset of Figure $1 \mathrm{c}$. The map indicates uniformity over a large area.

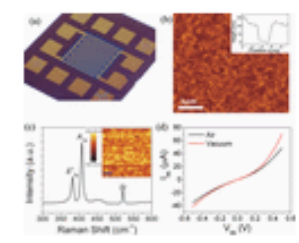

Figure 1. a) Photograph of a $\mathrm{MoS}_{2}$ thin-film with interdigitated electrodes. The blue area underneath the interdigitated patterns is the $\mathrm{MoS}_{2}$ channel. b) AFM image of the $\mathrm{MoS}_{2}$ film. The roughness is estimated to be $2.5 \mathrm{~nm}$ RMS. Inset: Line profile over a hole in the film, showing the thickness to be approximately $20 \mathrm{~nm}$. c) Raman spectrum of the $\mathrm{MoS}_{2}$ film. Inset: Scanning Raman map of the $A_{1 \mathrm{~g}}$ peak sum over a $10 \mu \mathrm{m} \times 10 \mu \mathrm{m}$ area. d) Source-drain current versus source-drain voltage $\left(l_{d s}-V_{d s}\right)$ characteristics of a $20 \mathrm{~nm}$ thick $\mathrm{MoS}_{2}$ film in air (black) and vacuum (red). Schematic of the vapor phase sulfurization technique. 
The source-drain current versus source-drain voltage $\left(I_{\mathrm{ds}}-V_{\mathrm{ds}}\right)$ characteristics are shown in Figure $1 \mathrm{~d}$. The $20 \mathrm{~nm}$ thick MoS $\mathrm{S}_{2}$ film exhibits non-linear behavior at a bias sweep in the range of $\pm 0.5 \mathrm{~V}$, implying the existence of a Schottky barrier between the metal contact and the semiconducting channel. The increased current level observed in vacuum indicates that adsorbates from atmosphere, e.g. water vapor, can be removed from the surface of the $\mathrm{MoS}_{2}$. Therefore, the films were first exposed to vacuum after loading into the gas sensing chamber.

The sensor performance can be evaluated in terms of sensor response $(S)$ and response time. In this study, $S$ is defined by the relative resistance change, as follows:

$$
S=\frac{\Delta R}{R_{0}}=\frac{R_{\text {Senox }}-R_{0}}{R_{0}} \times 100 \%
$$

where $R_{0}$ is the initial resistance of the sensor and $R_{\text {Sensor }}$ is the measured resistance upon gas introduction.

Figure 2a shows typical gas sensor response curves at various concentrations of $\mathrm{NH}_{3}$ gas, from 2 to $30 \mathrm{ppm}$ (parts-per-million) with a bias voltage of $0.5 \mathrm{~V}$. As $\mathrm{NH}_{3}$ is an electron-donor, it exhibits n-doping characteristics. When a $\mathrm{MoS}_{2}$ film is exposed to gaseous $\mathrm{NH}_{3}$, adsorbed molecules on the surface of $\mathrm{MoS}_{2}$ shift the Fermi level to the conduction band, resulting in a resistance decrease consistent with n-type behavior. The $\mathrm{MoS}_{2}$ film shows an immediate response, even though gases were introduced for only $15 \mathrm{~s}$. It is important to note, that the response is limited by the relatively high volume of our gas sensing chamber. The sensor also works at sub-ppm levels as shown in Figure $\underline{2} \mathrm{~b}$, as a clear signal is still visible as low as $300 \mathrm{ppb}$. The sensor sensitivity is linearly proportional to the concentration of $\mathrm{NH}_{3}$ introduced in the low concentration range (Figure 2 $\mathrm{c}$ ), which makes determination of gas concentration feasible. It is also visible that its recovery in pure $\mathrm{N}_{2}$ flow is not complete at room temperature, as its baseline gradually shifts during consecutive $\mathrm{NH}_{3}$ injections. This may be attributed to tight bonding between the $\mathrm{MoS}_{2}$ channel and $\mathrm{NH}_{3}$ molecules, as has been commonly mentioned for nanomaterial-based sensors with extremely high sensitivities. The recovery speed could potentially be accelerated by ultra-violet light illumination or annealing.[13-15]

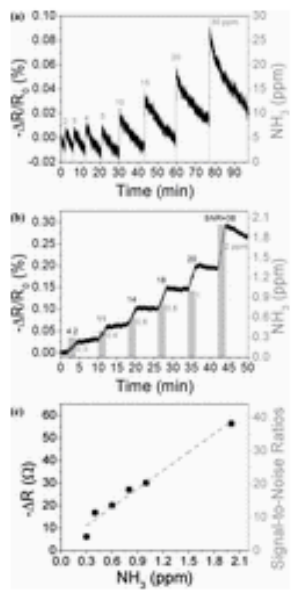

Figure 2. Sensor response plots show percentile resistance change versus time of the $\mathrm{MoS}_{2}$ film with a bias voltage of $0.5 \mathrm{~V}$, upon consequent $\mathrm{NH}_{3}$ exposures at various concentrations: a) from $2 \mathrm{ppm}$ to $30 \mathrm{ppm}$ and b) $300 \mathrm{ppb}$ to $2 \mathrm{ppm}$. The gray vertical bars indicate $\mathrm{NH}_{3}$ gas injections for: a) $15 \mathrm{~s}$ and b) 2 min, respectively. The signal-to-noise ratio (SNR) of each folding curve is shown to verify a sensible signal. c) Plots of resistance change (black solid circles) and signal-to-noise ratios (gray open boxes) as a function of $\mathrm{NH}_{3}$ concentration. The linear dashed line indicates the fitted line.

Our empirical limit of detection (LOD) has been measured as $300 \mathrm{ppb}$ with a signal-to-noise ratio (SNR) of 4.2. The initial resistance was calculated from the first 1000 data points before the first $\mathrm{NH}_{3}$ injection and the RMS noise was derived from the baseline. As the SNR must be at least three or larger for a viable sensor according to the IUPAC definition, [16] the theoretical LOD is extrapolated from the linearity of the SNR vs. $\mathrm{NH}_{3}$ concentration. Therefore, the outstanding SNR of our $\mathrm{MoS}_{2}$ film suggests that a detection threshold of $51 \mathrm{ppb}$ is achievable.

In previous reports on graphene gas sensors, the sensors produced have commonly been shown to be less sensitive to $\mathrm{NH}_{3}$ than $\mathrm{NO}_{x}$ because of weak binding (ca. $20 \mathrm{meV}$ ) and small charge transfer $(0.03 e)$ energy of $\mathrm{NH}_{3}$ compared to $\mathrm{NO}_{x}$. [17] Whilst mechanically exfoliated graphene has shown sensitivity in the ppb range upon $\mathrm{NO}_{2}$ exposure, only ppm levels of $\mathrm{NH}_{3}$ were detectable under the same conditions.[14] Likewise, similar results have been reported in preliminary studies of $\mathrm{MoS}_{2}$ sensors. [7, 8] Thus, our $\mathrm{MoS}_{2}$ sensors demonstrate a remarkable LOD for $\mathrm{NH}_{3}$, among the best reported for non-functionalized nanomaterial sensors, and may prove to be even more sensitive to other species. Future investigations will target other TMD films to achieve selectivity towards various gases.

We have presented vapor phase growth of $\mathrm{MoS}_{2}$ thin-films and investigated their performance as gas sensors for the detection of $\mathrm{NH}_{3}$. The thickness of $\mathrm{MoS}_{2}$ thin-films is easily controllable by modifying the thickness of pre-deposited Mo layers and the quality and uniformity of our thin-films has been demonstrated with Raman spectroscopy. $\mathrm{MoS}_{2}$ thin-film sensors show a fast response time towards $\mathrm{NH}_{3}$ with highly sensitive detection, however they don't immediately recover at room temperature. The LOD was experimentally achieved down to $300 \mathrm{ppb}$ and theoretical derivation indicates the potential for much higher sensitivity. In conclusion, we suggest that vapor phase grown $\mathrm{MoS}_{2}$ thin-films are strong candidates for chemical sensors and they have commercial potential due to the scalable, low-cost, high-yield production of the active channel, and their compatibility with conventional semiconductor fabrication processing. 
$\mathrm{MoS}_{2}$ thin-films were prepared by sulfurization of molybdenum (Mo) layers based on a vapor phase growth technique as shown in Figure $\underline{3}$. A thin layer of Mo $\left(99.99 \%\right.$, MaTecK) was deposited on a heavily doped p-type silicon (Si) substrate with $300 \mathrm{~nm}$ thick silicon dioxide $\left(\mathrm{SiO}_{2}\right)$ layer by sputtering using a Gatan PECS tool. A metal shadow mask was used to define the selective channel area. The Mo layer on the $\mathrm{Si} / \mathrm{SiO}_{2}$ substrate was placed in a quartz tube furnace and heated to $750^{\circ} \mathrm{C}$, with a ramping speed of $20^{\circ} \mathrm{C} / \mathrm{min}$, at an argon (Ar) flow of 150 sccm (standard cubic centimeters per minute), at a pressure of approximately 1 Torr. After 30 min annealing, sulfur (S) was introduced through a second upstream hot zone by heating sulfur powder $\left(99 \%\right.$, MaTecK) to its melting point $\left(113^{\circ} \mathrm{C}\right)$ for 15 min. The sulfur vapor reacted with Mo entirely, forming a thin $\mathrm{MoS}_{2}$ film before being cooled to room temperature under Ar flow.

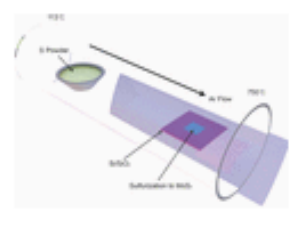

Figure 3. Schematic of the vapor phase sulfurization technique.

The $\mathrm{MoS}_{2}$ films were investigated by Raman spectroscopy using a Witec Alpha 300R confocal Raman microscope with a $532 \mathrm{~nm}$ laser wavelength and an 1800 lines per mm grating. A laser power of $<1 \mathrm{~mW}$ was employed in order to minimize sample damage. Scanning Raman mapping involved taking 4 scans per $\mu \mathrm{m}$ in both the $x$ and $y$ directions $(10 \mu \mathrm{m} \times 10 \mu \mathrm{m}, 1600$ spectra).

Gold electrodes were sputtered on top of an adhesion layer of titanium ( $\mathrm{T} / \mathrm{Au}=10 / 40 \mathrm{~nm}$ ) using a metal shadow mask and an interdigitated electrode (IDE) pattern (Figure 2a), which has a width-to-length ratio of 277 with a $200 \mu \mathrm{m}$ gap. This was used to increase the total current level as $\mathrm{MoS}_{2}$ crystals have a high resistivity.[18] AFM analysis was performed with a Vecco Dimension 3100.

For gas sensing tests, the $\mathrm{MoS}_{2}$ thin-film devices were placed on a custom-made chip-carrier and Au wires were electrically connected between binding pads by wire-bonding. All the samples were loaded in a gas sensing chamber at a pressure of $10 \mathrm{Torr}$ and $200 \mathrm{ppm} \mathrm{NH}_{3}$ gas, which was balanced with nitrogen $\left(\mathrm{N}_{2}\right)$, was used to introduce diluted $\mathrm{NH}_{3}$ gas with dry $\mathrm{N}_{2}$ carrying gas at a constant flow rate of 100 $\mathrm{sccm}$. The current change of $\mathrm{MoS}_{2}$ sensors upon interval gas exposure was measured at a constant bias voltage $(0.5 \mathrm{~V})$ at room temperature using a Keithley model 2612A SourceMeter.

[1] K. Mak, C. Lee, J. Hone, J. Shan, T. Heinz, Phys. Rev. Lett. 2010, 105, 136805.

[2] J. N. Coleman, M. Lotya, A. O'Neill, S. D. Bergin, P. J. King, U. Khan, K. Young, A. Gaucher, S. De, R. J. Smith, I. V Shvets, S. K. Arora,

G. Stanton, H.-Y. Kim, K. Lee, G. I. Kim, G. S. Duesberg, I. Hallam, J. J. Boland, J. J. Wang, J. F. Donegan, J. C. Grunlan, G. Moriarty, A. Shmeliov, R. J. Nicholls, J. M. Perkins, E. M. Grieveson, K. Theuwissen, D. W. McComb, P. D. Nellist, V. Nicolosi, Science 2011, 331, 568.

[3] B. Radisavljevic, A. Radenovic, J. Brivio, V. Giacometti, A. Kis, Nat. Nanotechnol. 2011, 6, 147.

[4] H. Liu, A. T. Neal, P. D. Ye, ACS Nano 2012, 6, 8563.

[5] S. Das, H.-Y. Chen, A. V. Penumatcha, J. Appenzeller, Nano Lett. 2013, 13, 100 .

[6] M. S. Fuhrer, J. Hone, Nat. Nanotechnol. 2013, 8, 146.

[7] H. Li, Z. Yin, Q. He, H. Li, X. Huang, G. Lu, D. W. H. Fam, A. I. Y. Tok, Q. Zhang, H. Zhang, Small 2012, 8, 63.

[8] Y. Yao, L. Tolentino, Z. Yang, X. Song, W. Zhang, Y. Chen, C. Wong, Adv. Funct. Mater. 2013, 23, 3577.

[9] K. Lee, H.-Y. Kim, M. Lotya, J. N. Coleman, G.-T. Kim, G. S. Duesberg, Adv. Mater. 2011, 23, 4178.

[10] Y. Zhan, Z. Liu, S. Najmaei, P. M. Ajayan, J. Lou, Small 2012, 8, 966.

[11] H. Li, Q. Zhang, C. C. R. Yap, B. K. Tay, T. H. T. Edwin, A. Olivier, D. Baillargeat, Adv. Funct. Mater. 2012, 22, 1385.

[12] X. Zhang, W. P. Han, J. B. Wu, S. Milana, Y. Lu, Q. Q. Li, A. C. Ferrari, P. H. Tan, Phys. Rev. B 2013, 87, 115413.

[13] J. Li, Y. Lu, Q. Ye, M. Cinke, J. Han, M. Meyyappan, Nano Lett. 2003, 3, 929.

[14] F. Schedin, aK. Geim, S. V Morozov, E. W. Hill, P. Blake, M. I. Katsnelson, K. S. Novoselov, Nat. Mater. 2007, 6, 652.

[15] G. Ko, H.-Y. Kim, J. Ahn, Y.-M. Park, K.-Y. Lee, J. Kim, Curr. Appl. Phys. 2010, 10, 1002.

[16] L. A. Currie, Pure Appl. Chem. 1995, 67, 1699.

[17] O. Leenaerts, B. Partoens, F. Peeters, Phys. Rev. B 2008, 77, 1.

[18] A. J. Grant, T. M. Griffiths, G. D. Pitt, A. D. Yoffe, J. Phys. Chem. C 1975, 17, L17-L23. 